\title{
Médiévales
}

Langues, Textes, Histoire

71 | automne 2016

Conflits et concurrence de normes

\section{Le statut du judaïsme dans le droit romain à la lumière du contentieux des conflits de lois $\left(\mathrm{IV}^{\mathrm{e}}-\mathrm{V}^{\mathrm{e}}\right.$ siècles)}

The Status of Judaism in Roman Law According to Legal Disputes Related to Conflicts of Laws (Fourth-Fifth Century)

\section{Capucine Nemo-Pekelman}

\section{QpenEdition}

\section{Journals}

Édition électronique

URL : https://journals.openedition.org/medievales/7878

DOI : 10.4000/medievales.7878

ISSN : 1777-5892

Éditeur

Presses universitaires de Vincennes

Édition imprimée

Date de publication : 20 novembre 2016

Pagination : 13-26

ISBN : 978-2-84292-565-9

ISSN : 0751-2708

\section{Référence électronique}

Capucine Nemo-Pekelman, « Le statut du judaïsme dans le droit romain à la lumière du contentieux des conflits de lois (Ive-ve siècles) », Médiévales [En ligne], 71 | automne 2016, mis en ligne le 20 septembre 2018, consulté le 22 avril 2022. URL : http://journals.openedition.org/medievales/7878 ; DOI : https://doi.org/10.4000/medievales.7878 
Capucine Nemo-Pekelman

\section{Le statut du judaïsme dans le droit romain à la lumière du contentieux des conflits de lois (IVe-Ve siècle)}

Les juristes de l'époque classique ne nous ont pas livré de textes de doctrine qui nous permettraient de comprendre en quels termes ils envisageaient les situations de coexistence juridique découlant de la présence dans les provinces de l'Empire romain de peuples ne s'organisant pas en communautés politiques mais possédant d'évidence des droits. Dans sa fameuse ouverture du manuel des Institutes (I, 1), le jurisconsulte Gaius expose que coexistaient en son temps - le II ${ }^{\mathrm{e}}$ siècle de notre ère - une pluralité de droits civils propres à chaque cité de l'Empire, mais il ne mentionne pas les droits qui existaient en dehors du cadre de la polis ou de la civitas, au sein de populations villageoises, tribales ou diasporiques. Ainsi en particulier ne possédons-nous pas d'écrits doctrinaux exposant comment était conçu le droit des populations juives qui vivaient en communautés diasporiques au sein des cités et villages des provinces romaines de l'Empire. Cependant, pour la période qui nous occupe ici - les $I^{\mathrm{e}}$ et $\mathrm{v}^{\mathrm{e}}$ siècles - il est des sources qui peuvent utilement nous renseigner ${ }^{1}$. Il s'agit de décisions impériales - dites « constitutions »- par lesquelles le pouvoir, alors partagé entre la chancellerie d'Occident et celle de Constantinople, dut trancher des conflits de lois et de juridictions entre les droits et tribunaux romains et juifs.

Ces textes ont un double intérêt. Principalement, ils nous disent quel fut le cadre juridique romain qui rendit possible, longtemps après que l'édit de Caracalla (212) eut octroyé le droit de cité romain aux pérégrins, la persistance du droit et des tribunaux juifs dans l'Empire. Secondairement, ils permettent de comprendre comment le pouvoir de l'époque définit ce que, de nos jours, nous appelons le judaïsme. À cet égard, il faut signaler

1. Les sources juridiques relatives aux juifs dans l'Empire romain sont recensées et commentées dans A. Linder, The Jews in Roman Imperial Legislation, Détroit/Jérusalem, 1987. 
l'extraordinaire intérêt - rarement souligné - des constitutions impériales. Un certain nombre de celles qui sont conservées dans le Code théodosien (438, désormais $C T h$ ) résultent de contentieux démarrés dans les provinces. Ces lois d'origine judiciaire - et celles que nous allons examiner maintenant en font partie ${ }^{2}$ - portent donc en elles la trace de débats et de négociations en justice, ce qui implique, et c'est l'intérêt que nous leur trouvons, que les justiciables juifs et leurs conseils (nomikoi, iurisperiti) participèrent à la recherche des solutions ${ }^{3}$. Ainsi ne paraît-il pas outré de dire que les termes désignant le judaïsme - leges, consuetudines, mores, religio ou superstitiofurent, jusqu'à un certain point, assumés par les juifs eux-mêmes, qui trouvèrent au terme d'un dialogue avec le pouvoir impérial les traductions romaines de réalités juridiques et institutionnelles juives.

L'origine casuistique et dialectique des constitutions interdit donc, selon nous, de les envisager au travers du seul prisme de l'idéologie impériale et, à plus forte raison, d'y lire une conception chrétienne des juifs et du judaïsme. C'est pourquoi nous nous distançons de la lecture qu'en a proposée Daniel Boyarin dans son ouvrage de 20044. Selon lui, les lois impériales, avec les écrits d'hérésiologues et historiens chrétiens contemporains, seraient symptomatiques «d'une mutation épistémologique de grande importance », laquelle aurait consisté à transformer le sens traditionnel du mot religio pour l'identifier à un système de croyances ${ }^{5}$. L'orthodoxie chrétienne, dont la voix se serait fait entendre dans les lois

2. Certaines décisions impériales ont été suscitées par des procédures par rescrits. Cette procédure précontentieuse permettait à un justiciable de requérir par supplique auprès de la chancellerie impériale un rescrit tranchant en droit son cas litigieux, lequel devait ensuite être porté devant le tribunal du gouverneur pour qu'il jugeât le litige en fait et de manière contradictoire. Un second type de procédure pouvait être déclenché pendant le procès, lorsque le juge confronté à une difficulté de droit saisissait pour éclaircissement la chancellerie d'une demande de rescrit ad relationem. $C f$. E. ANDT, La Procédure par rescrit (IVe-ve siècles), Paris, 1920.

3. Caroline Humfress souligne la nécessité qu'il y a à interpréter les sources judiciaires du point de vue des stratégies mises en œuvres par les litigants. $C f$. C. Humfress, « Law and Custom under Rome », dans A. Rio éd., Law, Custom and Justice in Late Antiquity and the Early Middle Ages, Londres, 2011, p. 23-49, ainsi que A. Dolganov, « Reichsrecht and Volksrecht in Theory and Practice : Roman Law and Litigation Strategy in the Province of Egypt », dans M. Jursa et H. TÄUBer éd., Administration, Law and Administrative Law : Comparative Studies in Imperial Bureaucracy and Officialdom, Vienne (à paraître).

4. D. Boyarin, La Partition du judaïsme et du christianisme, trad. J. Rastoin, Paris, 2011, p. 373-382.

5. Daniel Boyarin, à la suite d'Andrew Jacobs, Seth Schwartz, ou Susanna Elm, affirme que la notion de religion comme « catégorie à part de l'expérience humaine » fut inventée au tournant du IV et du $\mathrm{v}^{\mathrm{e}}$ siècles. Loin d'être un phénomène universel et anhistorique, elle serait donc un produit culturel chrétien de l'Antiquité tardive. De fait, «l'univers discursif » de cette période révélerait une réorganisation des "pratiques culturelles et marqueurs identitaires » - traditionnellement agencés autour de l'ethnos ou de la langue - sous les appellations nouvelles de "christianisme » et d' " hellénisme », ces nouvelles communautés se définissant désormais par leurs croyances. Le « judaïsme » serait aussi une invention de l'orthodoxie chrétienne triomphante, laquelle aurait cherché, pour mieux se définir, à identifier une seule 
impériales, aurait dès lors défini le judaïsme comme une religion alternative mais fausse ${ }^{6}$. Or, pour les raisons que nous avons dites, le choix de ces vocables fut largement contextuel, dépendant chaque fois de raisonnements adaptés à la recherche de solutions propres à des problèmes juridicopolitiques spécifiques ${ }^{7}$. En effet, les juristes ne mobilisaient pas les mêmes notions lorsqu'il s'agissait d'accorder des privilèges aux desservants de synagogues, de condamner pénalement des attaques de synagogues ou de régler des conflits de lois et de juridictions. Pour la même raison, nous sommes en désaccord avec l'idée selon laquelle le judaïsme aurait été défini comme une religio licita. La constitution impériale de 393 (CTh XVI, 8, 9) condamnant des attaques de synagogues qui sert de fondement à cette thèse - arbitrairement rapprochée de l'expression utilisée deux siècles plus tôt par Tertullien - ne saurait être considérée comme un texte représentatif à lui seul d'une conception romaine du judaïsme ${ }^{8}$.

La présente étude va donc consister à expliciter les logiques juridiques qui, à l'époque romaine tardive, permirent l'insertion ou le rejet du droit et des instances de jugement juifs dans l'ordre juridique romain. Elle permettra aussi, espérons-nous, de débrouiller les différents raisonnements qui présidèrent aux choix, en apparence anarchiques, des divers vocables utilisés par le législateur pour désigner le judaïsme. Avant de ce faire, on justifiera l'emprunt que nous faisons à des concepts juridiques modernes - un exercice périlleux mais qu'assument beaucoup d'historiens du droit - tels ceux, en l'occurrence, de « conflits de lois » et de « conflits de juridictions ».

« religion juive orthodoxe » représentée par le Patriarche de Galilée, en condamnant les autres sectes juives comme des hérésies.

6. Après Amnon Linder, Daniel Boyarin affirme qu'à compter d'une constitution impériale datée de 416, le judaïsme n'aurait plus été désigné que comme une superstitio. Cf. D. Boyarin, La Partition..., p. 377.

7. Andrew Jacobs concède que : «Les pratiques disciplinaires du droit romain, par exemple, fonctionnaient d'une manière tout à fait distincte de l'inculcation intellectuelle de l'historiographie ou de la mise en application ritualisée de l'orthodoxie. » Mais ceci ne l'empêche pas de conclure : « Le but commun de cet univers discursif était la réorganisation des aspects importants de la vie sous une unique rubrique chrétienne, totalisante, impériale » (cité par D. Boyarin, La Partition..., p. 353-354). Nous croyons au contraire que les constitutions impériales résultant d'un processus de raisonnement et d'élaboration à part ont un statut épistémologique original. Ainsi Jean-Pierre Coriat explique que, contrairement aux techniques législatives actuelles que nous connaissons par exemple en France, lesquelles, du moins en théorie, " consistent à déduire les solutions juridiques particulières de normes générales - traités, déclarations de droits, constitutions, lois - selon un système de réglementation dit hypothético-déductif », les méthodes d'élaboration du droit romain étaient « caractéristiques d'un système juridique dit axiologique ou casuistique, c'est-à-dire que la solution ne résultait pas d'une déduction syllogistique mais d'un débat contradictoire fondé sur l'argumentation et la controverse dialectique ». Cf. J.-P. CoRiat, Le Prince législateur, Rome, 1997, p. 457-458.

8. Sur l'usage abusif de la notion de religio licita, voir notamment L. V. Rutgers, « Roman Policy towards the Jews : Expulsions from the City of Rome during the First Century C. E. », Classical Antiquity, 13 (1994), p. 58-59. 


\section{Les notions de "conflit de lois " et de "conflit de juridictions " appliquées à l'Antiquité romaine tardive}

Les outils du droit international privé, plus précisément ceux de la « méthode conflictuelle », sont parfois utilisés pour analyser les situations de pluralisme juridique dans l'Empire romain ${ }^{9}$. Ainsi use-t-on de notions comme le «conflit de lois » ou le « conflit de juridictions » pour interpréter les phénomènes qui naquirent de la rencontre du droit de l'envahisseur romain avec les droits locaux des populations conquises. Ces notions modernes peuvent utilement éclairer la question, pour peu qu'elles soient maniées avec précaution.

Traduire, d'abord, les rapports entre les ordres juridiques impérial et locaux en termes de relations internationales peut paraître problématique dans la mesure où, habituellement, le droit international privé étudie les conflits entre systèmes juridiques d'États souverains et indépendants. Il est vrai que cette matière connaît aussi les conflits de lois et de juridictions à l'intérieur d'un État dont le système juridique n'est pas unifié, comme au sein d'un État fédéral ou même d'un État unitaire, et il est donc possible, a fortiori, d'y inclure l'Empire romain ${ }^{10}$. Mais alors, il convient d'avoir présent à l'esprit que les problématiques soulevées se situaient dans un contexte où les acteurs ne se plaçaient pas sur un pied d'égalité et où il revenait au pouvoir romain de déterminer souverainement ce qu'était une loi, une juridiction, et ce que devait être la solution du conflit.

Parler, ensuite, de conflits de « lois » nécessite des explications dans la mesure où le caractère juridique des normes sociales juives pourrait poser question. Certes il est depuis longtemps assez généralement entendu que l'on peut parler de droit sans État ${ }^{11}$. Anthropologues et sociologues

9. H. Cotton, « Private International Law or Conflicts of Laws : Reflections on Roman Provincial Jurisdiction », dans R. HAEnsch et I. HeINRICH éd., Herrschen und Verwalten. Der Alltag der römischen Administration in der Hohen Kaiserzeit, Cologne, 2007, p. 235-255 et H. LewALD, «Conflits de lois dans le monde grec et romain », Labeo, 5 (1959), p. 334-369. Voir aussi D. GAURIER, Histoire du droit international. De l'Antiquité à la création de l'ONU, Rennes, 2014.

10. J.-P. Laborde et S. Sana-Chaillé de Néré, Droit international privé, Paris, 2014, p. 102-103.

11. Les conceptions étatistes anciennes représentées par Raymond Carré de Malberg et Hans Kelsen ont, à partir des travaux du juriste hollandais Cornelis Van Vollenhoven (1901), été critiquées par les théoriciens du pluralisme juridique comme dénotant une conception moniste du droit teintée d'ethnocentrisme. Les penseurs pluralistes considèrent ainsi que des entités comme l'entreprise, le syndicat, la famille ou la communauté religieuse sont elles aussi créatrices de pouvoirs et de normes juridiques. Pour une synthèse de l'évolution de la notion de pluralisme juridique, $c f$. N. Rouland, Introduction historique au droit, Paris, 1998, p. 119122 ; H. Moutoun, « Pluralisme juridique », dans D. Alland et S. Rials éd., Dictionnaire de la culture juridique, Paris, 2003, p. 1158-1162, et G. R. Woodman, « Ideological Combat and Social Observation. Recent Debate about Legal Pluralism », The Journal of Legal Pluralism and Unofficial Law, 30-42 (1998), p. 21-59. Notons cependant que des auteurs continuent 
du droit proposent ainsi des définitions du droit non réductibles au cadre étatique. Ils estiment qu'une norme sociale peut être qualifiée de juridique dès lors qu'elle satisfait à deux critères (cumulés ou non, selon les écoles) : elle doit ouvrir la possibilité de sa transgression devant une instance de contestation, c'est-à-dire devant un tiers juge (critère de la justiciabilité) ; elle doit pouvoir éventuellement être imposée par une coercition extérieure et physique (critère de la contrainte) ${ }^{12}$. Or ce second critère paraît manquer pour ce qui concerne le droit juif dans l'Empire romain, lequel ne repose pas sur les outils de la coercition qui, s'ils existent, paraissent secondaires. Pour résoudre cette difficulté, on se contentera de rapporter la pensée de Robert Cover telle qu'elle a récemment été rappelée par Ando Clifford ${ }^{13}$. Robert Cover s'oppose à une conception du juridique considéré au prisme du contrôle social. Pour lui, seul « l'étudiant en droit peut en venir à identifier le monde normatif avec l'outillage professionnel du contrôle social ». Il distingue donc un univers « impérial », celui de « l'étudiant en droit », dans lequel les normes sont imposées par la force, et un univers qu'il désigne comme «païdéique » parce que « le nomos existe par la narration qui le situe et donne sens à ses prescriptions ». Dans les communautés païdéiques - l'auteur prend notamment pour exemple les communautés juives et chrétiennes américaines contemporaines - existe une force normative motrice découlant de liens interpersonnels forts et de significations communes, fondées sur des rituels, des prières partagées et, surtout, sur un corpus narratif commun - Torah ou Évangiles - enseigné, cru et reconnu. L'obéissance n'est alors que le corollaire de la compréhension ${ }^{14}$. Si nous faisons nôtres les arguments de Robert Cover, nous pouvons

de penser qu'il ne saurait exister de droit proprement dit, ni antérieurement à la création de l'État ni extérieurement à ce dernier, dans la mesure où le droit doit selon eux être considéré principalement sous la forme de la contrainte extérieure que seul serait en capacité d'infliger un pouvoir souverain. $C f$. J. CARBonnier, Sociologie juridique, Paris, 1978, p. 323-326 et, plus récemment, B. Z. TAmanAHA, « The Folly of the "Social Scientific" Concept of Legal Pluralism », Journal of Law \& Society, 20 (1993), p. 192-217.

12. Cette définition sommaire et simplifiée de ce qu'est la norme juridique, et qui constitue une synthèse du limpide exposé de Jean Carbonnier, suffit aux besoins de la présente étude. Cf. J. Carbonnier, Sociologie juridique..., p. 318-328. Mais il est bien évident que le droit est un objet qui se saisit difficilement. Ainsi que l'écrit le doyen Vedel lui-même : «Voilà des semaines et même des mois que je "sèche" laborieusement sur la question. [...] "Qu'est-ce que le droit ?" [...] me voilà déconcerté tel un étudiant de première année remettant copie blanche, faute d'avoir pu rassembler les bribes de réponses qui font échapper au zéro. » Cité par N. Rouland, Introduction historique au droit..., p. 20.

13. R. M. Cover, « The Supreme Court, 1982 Term. Foreword : Nomos and Narrative », dans Harvard Law Review, 97 (1983), p. 4-69. Ce très important article a fait l'objet d'une traduction et d'un commentaire par F. Michaut, « Norme et narration », dans Le Droit dans tous ses états à travers l'œuvre de Robert Cover, Paris, 2001. Pour une analyse de la définition du droit par Cover appliquée à la période romaine antique, $c f$. C. ANDo, « Pluralism and Empire, from Rome to Robert Cover », Critical Analysis of Law, 1 (2014), p. 1-22.

14. R. M. Cover, «The Supreme Court, 1982 Term... », p. 12-13. 
légitimement parler de « lois » et plus largement de « droit » juifs dans l'Antiquité romaine, même si ceci ne présage pas de la conception que les Romains eux-mêmes en avaient, laquelle constitue le sujet de la recherche à suivre ${ }^{15}$. Remarquons enfin que, selon une historiographie récente, les écrits juridiques - halakhiques - réunis dans la Mishnah, le Talmud de Jérusalem et le Talmud de Babylone, n'auraient, contrairement à ce que l'on croyait auparavant, longtemps connu qu'une diffusion limitée à certaines régions palestiniennes ${ }^{16}$. Si cela est vrai, nous ne possédons, au-delà des ancestrales lois mosaïques de la Torah, que peu de témoignages de ce qu'était le droit privé et pénal réglementant les rapports quotidiens des populations juives des $\mathrm{IV}^{\mathrm{e}}$ et $\mathrm{V}^{\mathrm{e}}$ siècles en dehors des cercles talmudiques, en Palestine et dans la diaspora ${ }^{17}$. Notons que si l'on devait s'en tenir à ce que nous en dit le droit romain, lequel n'évoque les lois juives qu'à propos de la circoncision, des interdits alimentaires, du droit matrimonial, du shabbat et des fêtes, nous pourrions être fondés à nous demander si les juifs des provinces n'étaient pas juridiquement acculturés (au droit romain, grec ou autre), formant, pour reprendre l'expression de Robert Cover, des « communautés insulaires » ne revendiquant, par rapport aux communautés civiques dans lesquelles ils vivaient, qu'un statut d'autonomie relative sur des aspects limités du droit mosaïque. Cependant la Mosaicarum et Romanarum legum collatio pourrait, par exemple, fournir un témoignage indirect de ce que fut le droit pénal des juifs de Rome au IV ${ }^{\mathrm{e}}$ siècle et la question demeure donc encore ouverte $^{18}$.

Dans l'expression « conflit de juridictions », l'usage du mot « juridiction » pour désigner les tribunaux juifs est aussi contestable, ce terme étant réservé aux instances de jugement publiques dans lesquelles

15. Nous adoptons dans cet article un certain parti pris, celui du «pluralisme partiel », lequel consiste à considérer qu'un droit non étatique ne saurait constituer une norme juridique pérenne que dans la mesure où il serait naturalisé et hiérarchisé par l'État, en l'occurrence l'Empire romain. Mais il va de soi que les populations juives elles-mêmes ont pu considérer de l'intérieur leurs règles comme juridiques en dehors de toute reconnaissance officielle. La question de la définition par les juifs de l'Empire de leurs normes sociales et de leurs instances de jugement est du ressort des historiens.

16. C. Hezser, The Social Structure of the Rabbinic Movement in Roman Palestine, Tübingen, 1997, p. 103-128.

17. Même en l'absence de témoignage, il paraît probable que les communautés juives développèrent un droit interprété de la Bible et adapté aux réalités des provinces romaines des $\mathrm{IV}^{\mathrm{e}}$ et $\mathrm{V}^{\mathrm{e}}$ siècles même si, selon Jean Carbonnier, la Torah «a démontré son aptitude à remplir seule l'office d'un corpus iuris ». Selon lui, «c'est un fait historique que la loi mosaïque a constitué le droit public et privé d'une société et même d'un État et qu'elle forme aujourd'hui la base du droit privé des communautés israélites dans les pays (ceux du Proche-Orient) qui admettent une pluralité de statuts personnels d'origine confessionnelle». Cf. J. CARBONNIER, « La Bible et le droit », dans La Révélation chrétienne et le droit, Paris, 1961, p. 115-129.

18. Je remercie Samuele Rocca de m'avoir indiqué ce texte comme une hypothétique source juridique juive non talmudique. Sur la collatio, $c f$. V. Reutgers, The Jews in Late Ancien Rome, Leyde/New York/Cologne, 1995, p. 213-256. 
des représentants de l'État jugent en son nom ${ }^{19}$. Aussi préférerons-nous les appellations génériques et qui nous paraissent plus neutres de « tribunal » ou de « cour ». Il convient de préciser qu'existaient, pour les Juifs de l'époque tardo-antique, une pluralité de fora. Ils pouvaient, en théorie, attraire leurs défendeurs devant un tribunal public de droit commun ou spécialisé, au niveau, selon la nature de l'affaire, de la cité, de la province, de la préfecture du prétoire ou de la chancellerie impériale ${ }^{20}$. Nous savons ainsi que des justiciables juifs saisirent les chancelleries de Ravenne et de Constantinople par le biais d'une procédure précontentieuse dite "procédure par rescrit », preuve que certains d'entre eux pouvaient employer ces voies de droit techniques et coûteuses ${ }^{21}$. Nous savons également, par le témoignage d'Augustin d'Hippone, que des Juifs de sa cité eurent recours à l'audience de son tribunal épiscopal ${ }^{22}$. Quant aux tribunaux juifs, il s'en trouvait probablement, si l'on suit Martin Goodman, dans les communautés villageoises de Galilée ${ }^{23}$. D'autres tribunaux juifs relevaient du cadre communautaire de la synagogue. La fonction de juger les litiges figurait de fait parmi les très nombreuses activités assumées par la communauté synagogale et, selon Lee I. Levine, il est probable qu' un tribunal se réunissait à l'intérieur même de l'édifice ${ }^{24}$. Mais des Juifs pouvaient préférer avoir recours à des arbitres privés. Une constitution du 3 février 398 (CTh II, 1,2 ), sur laquelle nous reviendrons, nous apprend en effet que de simples particuliers pouvaient jouer le rôle de tiers arbitres, et Catherine Hezser a recensé, dans la Mishnah et le Talmud, des circonstances dans lesquelles des sages tannaim (rabbins des $\mathrm{I}^{\mathrm{er}}$ au $\mathrm{III}^{\mathrm{e}}$ siècle) et amoraim ( $\mathrm{IV}^{\mathrm{e}}-\mathrm{VI}^{\mathrm{e}}$ siècle), pour certains dépourvus de fonctions officielles dans le village et la synagogue, furent sollicités à ce titre ${ }^{25}$. La même constitution de 398 indique également que de mystérieux «patriarches » pouvaient aussi être juges. L'emploi du pluriel interdit selon nous d'identifier ces patriarches avec le Patriarche

19. E. Jeuland, Le Droit processuel, Paris, 2007, p. 101-106.

20. C. Humfress, "Thinking through Legal Pluralism : "Forum Shopping" in the Later Empire », dans J. Duindam, J. Harries, C. Humfress et N. Hurvitz éd., Law and Empire. Ideas, Pratices, Actors, Leyde/Boston, 2014, p. 234-244.

21. Cf.n. 2.

22. N. LENSKI, «Evidence for the Audientia episcopalis in the New Letters of Augustine », dans R. Mathisen éd., Law, Society, and Authority in Late Antiquity, Oxford, 2001, p. 83-97.

23. M. Goodman, State and Society in Roman Galilee. AD. 132-212, Totowa (NJ), 1982, chap. 10 .

24. Plus encore que les temples polythéistes, la synagogue antique était un lieu multifonctionnel de réunion, de prière et de repas, de récolte et de conservation de fonds, une école élémentaire, primaire et pour adultes, une bibliothèque et un dépôt d'archives, un lieu de résidence et de célébration (mariage, enterrement), et un tribunal. $C f$. L.I. LevinE, The Ancient Synagogue, New Haven/Londres, 2000, p. 370-386.

25. Si certains rabbins endossaient des rôles dans la synagogue, certains autres officiaient en dehors des cercles communautaires. Cf. C. Hezser, The Social Structure ..., p. 353-404 et H. LAPIN, Rabbis as Romans, Oxford, 2012, p. 98-125. 
Gamaliel V (et VI) de Galilée ${ }^{26}$, et il est possible que ce titre ait été porté par des primats qui, sans se situer dans une hiérarchie institutionnelle, laquelle paraît ne pas avoir existé contrairement à ce qui se passait dans l'Église chrétienne contemporaine, pouvaient occasionnellement juger des litiges se situant à un niveau "supra-synagogal ${ }^{27}$. De même, nous savons que le Patriarche de Galilée jugeait en son tribunal ${ }^{28}$. Enfin, probablement les deux sanhédrins qui, semble-t-il, avaient juridiction sur les populations juives des provinces de Palaestina Prima et Secunda endossaient-ils aussi occasionnellement un rôle judiciaire ${ }^{29}$.

\section{Les conflits de lois entre droit romain et lois juives}

Il s'agit de nous demander ce qui se passait lorsqu'un for romain se trouvait devoir trancher un litige comportant des éléments d'extranéité liés au fait que les litigants - du moins l'un d'entre eux - revendiquaient l'application d'une loi juive contredisant le ius commune.

Dans une première décision du 25 juillet 404 (Cth XVI, 8, 17), la chancellerie de l'empereur Honorius, saisie d'un litige opposant des membres d'une communauté synagogale à ses dirigeants, autorise les seconds à lever une taxe en faveur de discipuli envoyés annuellement de Galilée par le Patriarche Gamaliel. Il ne s'agit pas ici d'entrer dans le détail de cette passionnante affaire révélant les difficultés rencontrées par le Patriarche pour imposer son autorité sur les Juifs de la partie occidentale. Ce qui nous intéresse est la justification avancée dans la réponse impériale au soutien de l'autorisation de cette levée, laquelle est consentie au nom du respect dû à la « coutume » (consuetudo) des Juifs. Le second exemple résulte d'une constitution impériale de juillet 412 (Cth xvI, 8, 20 et 26), elle aussi issue de la chancellerie ravennate. Il semblerait qu'à l'origine de l'affaire, un défendeur juif avait refusé d'obéir à une citation en justice, ce qui constituait une violation du in ius vocatio, lequel obligeait la partie citée à promettre de comparaître à jour dit. Le Juif avait, pour justifier son refus, invoqué le respect dû au jour chômé du shabbat. La chancellerie, saisie probablement pour avis par le gouverneur de province juge de l'affaire, donne droit au défendeur juif, ce qu'elle justifie par la nécessité de respecter

26. Le pluriel pourrait certes aussi désigner, puisque la fonction du Patriarche de Galilée était dynastique, le père et le fils. C'est l'explication préconisée par Amnon Linder qui relève, un détail certes troublant, qu'en dehors des constitutions impériales aucune source - épigraphique, archéologique, littéraire juive ou non juive - ne mentionne l'existence de ces «petits patriarches », selon l'expression de Jean Juster. $C f$. A. LindER, The Jews..., p. 130, n. 12.

27. L'existence de « conciles provinciaux » juifs est mentionnée en 363 par l'empereur Julien. $C f$. A. Linder, The Jews..., p. 158, n. 3.

28. CTh XVI, 8, 22 : A. LindER, p. 269.

29. CTh XVI, 8, 29 : A. Linder, p. 320. 
«l'ancien usage » et la « coutume » des Juifs, auquel elle ajoute un argument de modération. Le dernier exemple tranche avec les premiers, raison pour laquelle nous le mentionnons à la fin, en dépit de la chronologie. Il s'agit d'une constitution impériale du 30 décembre 393 (CJ I, 9, 7) rendue depuis la chancellerie de Constantinople. La constitution censure cette fois la loi juive, ordonnant qu'aucun juif «ne conservera sa coutume» (morem suum) en matière d'union matrimoniale " ni ne choisira ses noces selon sa loi (legem suam) ni ne fera plusieurs mariages dans le même temps $»^{30}$.

L'évidente indifférence avec laquelle le pouvoir romain opte pour les termes de consuetudo, de mos, d'usus ${ }^{31}$, ou pour celui de lex constitue une source réelle de difficulté. En effet, depuis les travaux de Joseph MélèzeModrzejewski, il est courant de dire que le mécanisme juridique ayant permis la survivance des droits des anciens pérégrins avant et après 212 résulterait de ce que ces droits étaient intégrés dans l'ordonnancement juridique romain en tant que coutumes ${ }^{32}$. Les coutumes pouvaient en effet, sous les Sévères, désigner les lois des provinciaux, et si cet usage est à notre époque plus rare - mos et consuetudo faisant dans une proportion écrasante référence à des sources administratives ${ }^{33}-$, il en demeure quelques occurrences ${ }^{34}$. Comme, selon Joseph Mélèze-Modrzejewski, les coutumes se situaient à un rang normatif inférieur à celui des lois, le pouvoir romain pouvait décider de les intégrer en qualité de mos regionis, ou de les rejeter. C'est que l'autorité de ces coutumes locales n'était reconnue, selon une constitution de Constantin de 319, que pour autant qu'elles respectassent la raison et le droit écrit. La victoire du référent romain sur le référent juif aurait donc juridiquement été rendue possible au nom d'un principe de hiérarchie des normes.

Mais, s'il est vrai que les juristes de la chancellerie raisonnèrent bien en ces termes, pourquoi alors qualifièrent-ils les normes matrimoniales juives de « lois » pour, qui plus est, les censurer ? Récemment, José Luis Alonso a mis en cause cette théorie de la coutume provinciale en rappelant un consensus ancien selon lequel, à la différence de nos ordres juridiques

30. A. Rousselle, «Vivre entre deux droits : la pratique familiale poly-juridique des citoyens romains juifs », Annales ESC, 45 (1990), p. 839-859, et C. Nemo-Pekelman, Rome et ses citoyens juifs, Paris, 2010, p. 57-69.

31. Sur les nuances entre ces trois usages, $c f$. J. GAUDEMET, « La coutume au Bas-Empire. Rôle, pratique et notion théorique », Labeo, 2 (1956), p. 148-151.

32. J. Mélèze-ModrzeJewsKi, « Diritto romano e diritti locali », dans Storia di Roma III : l'età tardoantiqua, Turin, 1993, p. 985-1009.

33. CTh XII, 5, CJ XI, 63, 1 ; VIII, 52, 2 ; IV , 62, 4.

34. Sur la coutume, $c f$. L. Bove, « La consuetudine nell'evoluzione del diritto romano », dans « La coutume I », Recueil de la Société Jean Bodin 51, Bruxelles, 1990, p. 91-96 ; J. GAudEMET, «L'autorité de la loi et de la coutume dans l'Antiquité », dans Rapports généraux au $6^{e}$ congrès international de droit comparé, Bruxelles, 1964, p. 9-36 ; ID.. « La coutume au Bas-Empire... », p. 147-159. 
actuels, le système juridique romain ignorait toute hiérarchie des normes ${ }^{35}$. José Luis Alonso rappelle que la coutume n'a jamais été située à un rang inférieur mais, ce qui est fort différent, qu'elle s'imposait dans l'hypothèse du silence de la loi. Il propose une explication alternative à celle de Joseph Mélèze-Modrzejewski pour décrire le mécanisme juridique ayant permis la survivance des droits locaux. Suivons son raisonnement. Entre le milieu du $\mathrm{II}^{\mathrm{e}}$ siècle avant notre ère et le $\mathrm{III}^{\mathrm{e}}$ siècle, le droit privé était principalement de source prétorienne, le prêteur ayant, dans son tribunal, pouvoir de dire le droit en vertu de sa iurisdictio, et se départissant pour ce faire constamment du ius civile. Hors de Rome, les gouverneurs agissaient de même. Ayant pouvoir discrétionnaire de iurisdictio, ils n'étaient tenus par aucun principe de hiérarchie des normes, n'étant contraints par aucune d'elles, pour peu qu'aucun argument d'aequitas ou d'utilitas ne les y incite. En d'autres termes, poursuit-il, les Romains, contrairement aux juristes modernes, n'envisageaient pas les règles de droit du point de vue de leur force coercitive - potestas - mais comme étant simplement revêtues d'auctoritas. Or, les traditions, usages et coutumes, souvent invoqués au soutien du respect des droits indigènes - et les constitutions mentionnées plus haut rappellent à l'envi l'ancienneté des coutumes juives - étaient considérés comme sources d'auctoritas par le tribunal du gouverneur. Les juristes auteurs des trois constitutions susmentionnées auraient donc considéré les normes juives comme de véritables sources du droit mais revêtues, évidemment, d'une auctoritas moindre, raison pour laquelle ils n'auraient pas hésité, lorsqu'elles heurtaient leurs propres conceptions du droit et de la morale, à les censurer.

Soulignons enfin que ces conflits de lois se résolvaient selon un mode dit substantiel, c'est-à-dire qu'ils donnaient lieu à la création de nouvelles règles. En 393 était ainsi créée une incrimination sanctionnant les mariages endogames et bigames des Juifs, et par les constitutions de 404 et 412 étaient octroyées des exemptions au droit commun sous forme de privilegia. Remarquons que ces privilegia ne constituaient pas des droits mais des faveurs accordées par les empereurs intuitu personae, qui ne liaient pas leurs successeurs ${ }^{36}$. Le pouvoir avait de fait tout intérêt à délivrer des privilèges, une pratique qui lui permettait de s'assurer la clientèle de ses sujets $^{37}$. Il convient donc d'avoir à l'esprit la nature fragile et réversible du mécanisme d'intégration des lois juives dans l'ordre juridique romain.

35. J. L. Alonso, "The Status of Peregrine Law in Egypt: "Customary Law" ", The Journal of the Juristic Papyrology : Proceedings of the 27th Congress of Papyrology Keynote Papers, 43 (2016), p. 351-404.

36. C. Nemo-Pekelman, Rome..., p. 21-24.

37. Cf. E. Meyer-Zwiffelhoffer, Politikōs archein, Stuttgart, 2002, p. 172-222, qui rassemble des preuves du langage patronal concernant les normes romaines dans les provinces orientales. 


\section{Les conflits de compétence entre tribunaux juifs et juridictions romaines}

Il faut ici se représenter la situation très concrète du Juif citoyen romain usant de sa double appartenance pour s'adonner au forum shopping ${ }^{38}$. Frappé d'une sentence juive désavantageuse ou l'anticipant (parce que la solution juive et/ou les juges communautaires lui seraient en l'espèce défavorables), il attrait son contradicteur devant une juridiction publique. Le cas s'est sans doute présenté plusieurs fois et a parfois incité le juge romain, invité à se prononcer sur sa compétence, à saisir pour avis le pouvoir central. C'est dans ces circonstances que nous trouvons une décision donnée de Constantinople à la fin du IV siècle.

En 392 (CTh XVI, 8, 8), des Juifs bannis par « les primats de leur Loi » (legis primates) décident de demander l'annulation de la sentence, qu'ils obtiennent de la part de la juridiction du gouverneur de province entouré de ses assesseurs. Le tribunal synagogal désavoué interjette appel devant l'auditorium du préfet du prétoire d'Orient, lequel demande l'avis de Constantinople qui juge la plainte des juifs bannis irrecevable au motif que les tribunaux romains n'étaient pas compétents, les primats ayant « pouvoir de rendre des sentences en matière religieuse ${ }^{39} »$.

Cette solution, précise le texte, a été obtenue après consultation des « clarissimes et illustres patriarches », et il faut nous imaginer des notables juifs appartenant à la classe sénatoriale, secondés par des jurisconsultes ${ }^{40}$. Or la constitution de 392 quitte le vocabulaire du droit pour désigner le judaïsme comme une religio. Daniel Boyarin attribue ce changement de registre à l'influence des chrétiens orthodoxes alors puissants au sein de la chancellerie, lesquels auraient souhaité créer des « religions », païenne et juive, par rapport auxquelles situer le christianisme ${ }^{41}$. Selon nous, ce choix s'explique plutôt par la logique du précédent couramment mobilisée, comme on sait, dans le raisonnement casuistique. Ainsi en 330 (CTh XVI,

38. L'expression est de Caroline Humfress dans, notamment, « Thinking through Legal Pluralism... », p. 244. Elle désigne le phénomène consistant, pour des justiciables placés sous la juridiction de tribunaux concurrents (dans l'hypothèse, par exemple, où un tribunal serait compétent en raison du territoire et le second en raison de la personne) à opérer des choix stratégiques les conduisant à saisir le for devant lequel ils croient pouvoir obtenir la décision de justice la plus favorable.

39. CTh XVI, 8, 8 : «[...] quos virorum clarissimorum et inlustrium patriarcharum arbitrio manifestum est habere sua de religione sententiam [...] ». Pour une description de cette affaire, cf. C. Nemo-Pekelman, « Pouvoir et réseaux des juges juifs », dans A. Bodin et T. Moreau éd., Réseaux sociaux et contraintes dans l'Antiquité tardive, Revue des études tardo-antiques, suppl. 1 (2014), p. 289-306 [en ligne] : http://www.revue-etudes-tardoantiques.fr/wp-content/uploads/2014/07/Nemo-Pekelman.pdf [consulté le 5 juillet 2016].

40. Si nous acceptons, bien sûr, l'hypothèse que ces patriarches n'étaient pas ceux de la dynastie hillélite, dont il est établi qu'ils étaient illustres.

41. $C f$. n. 5 . 
8,2 ), des notables de communautés synagogales, pour obtenir en faveur de leurs desservants l'exemption des charges (munera) que faisaient peser sur les citoyens des villes les curies municipales, avaient plaidé que la synagogue accomplissait des fonctions « religieuses » et réclamé les mêmes privilèges que ceux des clergés polythéistes et chrétiens. À la fin du siècle, une constitution de 397 (CTh XVI, 8, 13) indique aussi que l'exemption doit être accordée à ceux qui se consacrent à cette « religion » ${ }^{42}$. Ainsi les synagogues étaient-elles, pendant le $\mathrm{IV}^{\mathrm{e}}$ siècle, considérées par Constantinople comme des associations religieuses, raison pour laquelle les juifs et leurs conseils rappelèrent cette qualification pour réclamer la reconnaissance de la compétence du tribunal synagogal en la matière.

Cependant, la solution consistant à dégager un critère de répartition rationae materiae entre les compétences juive et publique n'était pas effective, la matière religieuse n'ayant pas de frontières nettes. C'est si vrai que, dès l'année suivante, une constitution de 393 déjà mentionnée criminalisait le droit matrimonial juif. C'est dans un même esprit de défiance vis-à-vis du droit juif que, par une loi de 398 , Constantinople indiqua que les Juifs « vivant en Romains selon le droit commun » devaient «s'adresser aux tribunaux de la façon régulière [...] pour les causes relevant des procès, des lois et du droit, et que tous devaient engager des actions et se défendre selon le droit romain ${ }^{43}$. La décision précisait que les juges juifs, patriarches comme simples particuliers, ne pouvaient être que des arbitres privés. Les effets étaient importants car les autorités communautaires ainsi rappelées à l'ordre se voyaient interdire la possibilité de forcer un défendeur à comparaître devant elles, l'arbitrage reposant sur le volontariat. Plus que cela, elles ne pouvaient seules assurer l'exécution forcée de leurs sentences mais devaient entamer une procédure devant un tribunal public. Ainsi le refus de reconnaitre aux tribunaux juifs le privilège d'autonomie juridictionnelle nous paraît-il net.

Enfin, le moyen utilisé pour encadrer strictement, à défaut de pouvoir l'interdire, le pouvoir des juges juifs, consista à habiller les cours en instances d'arbitrage romaines par le moyen de la fiction juridique ${ }^{44}$. La loi de 398 indique que les juges traditionnels juifs seront saisis « comme s'ils étaient des arbitres » (ad similitudinem arbitrorum), c'est-à-dire « par compromis » (per compromissum) et, en toute hypothèse, «par accord conjoint »

42. Mentionnons aussi pour l'Occident la loi de Valentinien I ${ }^{\text {er }}$ définissant la synagogue comme un local « religieux » $(C T h$ vII, 8,2$)$.

43. Pour des commentaires détaillés de cette loi, $c f$. J. HARRIES, « Creating Legal Space. Settling Disputes in the Roman Empire », dans C. Hezser éd., Rabbinic Law in its Roman and Near Eastern Context, Tübingen, 2003, p. 63-82, et C. Nemo-Pekelman, Rome ..., p. 69-75.

44. Notons que ce mécanisme, dès l'origine, avait précisément été développé dans le but d'intégrer au ius civile des institutions conceptuellement étrangères. Sur cette question et sur l'usage, en particulier, de la fiction en matière de juridictions provinciales, $c f$. C. Ando, L'Empire et le Droit. Invention juridique et réalités historiques à Rome, Paris, 2013, p. 17 et p. 27-37. 
(ex consensu) des parties. Dans l'hypothèse où le débiteur du jugement refuserait de l'exécuter spontanément, la juridiction provinciale pourrait être impliquée. Mais sur quel fondement juridique ? En effet, l'arbitrage romain ex compromisso supposait, précisément, la réalisation en amont d'une convention assortie d'une double stipulation de peine ouvrant, contre celui qui contesterait l'arbitrage rendu, l'action née de la stipulation et le condamnant à régler le montant prévu de la poena. En l'absence, prévisible, de réalisation par les litigants juifs d'un tel document, le législateur recourt à une seconde fiction en précisant que la décision arbitrale pourra tout de même recevoir force exécutoire "comme si l'arbitre avait été affecté par sentence d'un juge », une autre forme d'arbitrage romain résultant de la nomination comme arbitre d'un iudex delegatus par le gouverneur-juge ${ }^{45}$.

En somme et pour conclure, le cadre juridique ayant permis que certaines lois juives puissent être autorisées et d'autres censurées consista à reconnaître à celles-ci la qualité de véritables sources du droit - coutumes ou lois - mais simplement revêtues d'une auctoritas que l'empereur, souverain en matière législative, pouvait admettre ou rejeter. Celles qui étaient officiellement reconnues l'étaient par voie de privilegia, ce qui rendait particulièrement réversible cette reconnaissance. Mais la chancellerie s'attaqua surtout aux tribunaux juifs, refusant de leur reconnaître tout pouvoir puisqu'elle les assimila, par fiction, à des instances d'arbitrage romaines, ce qui signifiait que les juges traditionnels ne pouvaient être saisis que sur un mode exclusivement volontaire. Or, un droit ne saurait survivre sans tribunaux susceptibles d'en assurer la sanction. Ainsi les réflexions de Clifford Ando à propos de la période du Haut-Empire, qui critique l'idée répandue selon laquelle l'Empire romain, contrairement aux États-nations modernes, aurait fourni un cadre juridico-politique exemplaire pour le respect du pluralisme juridique, nous paraissent-elles se vérifier pour ce qui concerne le traitement des provinciaux juifs de l'époque tardive ${ }^{46}$.

Pour ce qui regarde, enfin, la définition que donnèrent du judaïsme les lois romaines, les acteurs de l'époque - autorités impériales d'une part, litigants juifs et leurs conseils d'autre part - le caractérisèrent chaque fois

45. Pour Jill Harries, la désignation d'un arbitre par le gouverneur serait un vestige de la procédure civile ordinaire dans laquelle le choix du juge-arbitre chargé de trancher le litige était effectué par le préteur ou gouverneur de province. $C f$. J. HARrIEs, « Creating Legal Space... », p. 74-75. Nous pouvons également rapprocher la solution imaginée dans la loi de 398 de celle qui consistait, pour que les contrats des provinciaux puissent créer des effets exécutoires en justice, à recourir à une clause stipulatoire. $C f$. J. MÉLÈZE-ModrZEJEwSKI, « Loi et coutume dans l'Égypte grecque et romaine. Les facteurs de formation du droit en Égypte d'Alexandre à la conquête arabe », dans Journal of Juristic Papyrology, suppl. 21, Varsovie, 2014, p. 326.

46. C. Ando, « Pluralism and Empire... », p. 1-7 notamment. 
en fonction de la solution recherchée pour le cas d'espèce. Ainsi, lorsqu'il s'agit de revendiquer une autonomie juridictionnelle, les communautés invoquèrent leur qualité d'associations « religieuses ». Dans les litiges relatifs aux conflits de normes entre la loi mosaïque et le droit commun, le judaïsme fut plutôt présenté comme un corpus iuris, une Lex ancienne et vénérable. Ainsi s'explique le caractère largement erratique des terminologies utilisées, lesquelles se rebellent à toute lecture téléologique.

\section{Capucine Nemo-Pekelman - Université Paris-Ouest - EA CHAD}

\section{Le statut du judaïsme dans le droit romain à la lumière du contentieux des conflits de lois (IVe-Ve siècles)}

Aux IV et $V^{e}$ siècles, les populations juives réparties en foyers de peuplement dans de nombreuses provinces orientales et occidentales de l'Empire romain fournissent un exemple type d'entités diasporiques ne fonctionnant pas selon un modèle civique. Ceci n'empêcha pas le pouvoir romain de qualifier leurs règles, construites autour des prescriptions de la Torah, de leges ou de consuetudines, ce qui prouve qu'il leur reconnut la qualité de véritables sources du droit. Néanmoins, les lois impériales qui conservent la trace de litiges relatifs à des conflits de lois ou de juridictions suggèrent que, du point de vue romain, les lois juives étaient revêtues d'une auctoritas que l'empereur, souverain en matière législative, pouvait admettre ou rejeter. En outre, quand elles étaient formellement autorisées, c'était en qualité de simples privilèges. Les tribunaux juifs quant à eux (principalement les cours communautaires des synagogues et des villages) ne reçurent pas d'autonomie juridictionnelle mais furent assimilés, par fiction juridique, à des cours d'arbitrage romaines. L'étude entend aussi débrouiller les différents raisonnements qui présidèrent aux choix, en apparence anarchiques, des divers vocables - us, mos, consuetudo, lex, religio, superstitio - utilisés dans les lois impériales pour désigner le judaïsme.

Droit romain - droit juif - conflit de lois - conflit de juridictions - Empire romain tardif

\section{The Status of Judaism in Roman Law According to Legal Disputes Related to Conflicts of Laws (Fourth-Fifth Century)}

During the fourth and the fifth centuries, Jewish populations were scattered over settlements in many Eastern and Western provinces of the Roman Empire. All together, they formed a diasporic entity deprived of any civic organization - a sub-political community. That did not prevent the Roman legislator from qualifying their norms, based on the prescriptions of the Torah, as leges or consuetudines. This shows that they were officially recognized as legal sources. But Imperial laws, which kept track of legal disputes relating to conflicts of laws or jurisdictions, suggest that, from the Roman point of view, Jewish Laws carried an auctoritas that the Roman emperor, as a sovereign legislator, could admit or reject. Besides, when they were formally accepted, it was only as privileges. Jewish courts (mainly the communal courts in villages and synagogues) were not given any judicial autonomy. Quite to the contrary, through legal fiction, they were regarded as Roman courts of arbitration. The article also aims at disentangling what in Imperial Laws is seemingly a chaotic choice of vocabulary used as refer to Judaism (us, mos, consuetudo, lex, religio, and superstitio).

Roman Law - Jewish Law - Conflict of Laws - Conflict of jurisdictions - Late Roman Empire 